\title{
Ground-state properties of the two-dimensional charged Bose gas
}

\author{
B. Davoudi, ${ }^{1}$ E. Strepparola, ${ }^{2}$ B. Tanatar,${ }^{3}$ and M. P. Tosi ${ }^{2}$ \\ ${ }^{1}$ Institute for Studies in Theoretical Physics and Mathematics, Tehran 19395-5531, Iran \\ ${ }^{2}$ Istituto Nazionale di Fisica della Materia and Classe di Scienze, Scuola Normale Superiore, I-56126 Pisa, Italy \\ ${ }^{3}$ Department of Physics, Bilkent University, Bilkent, Ankara 06533, Turkey
}

(Received 3 April 2000; revised manuscript received 11 September 2000; published 13 February 2001)

\begin{abstract}
We study the two-dimensional gas of charged bosons interacting via a logarithmic potential within the quantal hypernetted-chain approximation. The pair correlation function, the static structure factor, the groundstate energy, and the spectrum of collective excitations are calculated over a range of the density parameter $r_{s}$. We find that the static and dynamical properties are described rather well in this approach in comparison to previous theories. Our results are in good agreement with the available quantum Monte Carlo simulations.
\end{abstract}

DOI: 10.1103/PhysRevB.63.104505

PACS number(s): 67.40.Db, 05.30.Jp, 71.45.Gm

\section{INTRODUCTION}

Two-dimensional (2D) electronic systems have attracted tremendous attention in the last two decades, and are the subject of continuing interest. ${ }^{1,2}$ The Coulomb interaction potential and the many-body effects it induces play an important role in determining the ground-state properties. The application of a 2D electron gas model in describing semiconductor heterojunctions and various other structures has proved quite useful for our understanding of correlation effects in low-dimensional systems. In the artificially fabricated semiconducting materials the underlying Coulomb interaction has a three-dimensional character. The Coulomb potential between point charges in a strictly $2 \mathrm{D}$ space, on the other hand, is obtained from the solution of the Poisson equation (in 2D) to give logarithmic behavior, viz., $\ln r$, in contrast to the $1 / r$ dependence encountered in 3D. The logarithmic potential has been utilized to develop a successful theory ${ }^{3}$ of defects and phase transitions mediated by them in $2 \mathrm{D}$ systems. The quantum aspects of $2 \mathrm{D}$ charged particles are relatively less studied. In this paper we investigate some ground-state properties of charged bosons interacting with a $\ln r$ potential. The model of a charged Bose fluid has been gaining attention in connection with high- $T_{c}$ superconductors, ${ }^{4}$ and our study should provide further insight to the correlation effects ${ }^{5}$ in these systems.

The ground-state correlations in 2D charged systems interacting via the $\ln r$ potential have been studied with a variety of methods. Calinon et $a .^{6}{ }^{6}$ investigated the properties of a classical one-component plasma (OCP) using the approximation technique of Singwi, Tosi, Land, and Sjölander ${ }^{7}$ (STLS). Bakshi et $a .^{8}{ }^{8}$ employed the convolution approximation for the classical OCP, and found lower ground state energies as a function of the coupling strength. Monte Carlo and molecular dynamics simulations of the classical system were also presented. ${ }^{9}$ Quantum 2D electron gas studies were presented by Thakur and Pathak ${ }^{10}$ within the STLS. The static and dynamic quantities of physical interest showed qualitatively similar results to the 3D case. Dharamvir and Pathak ${ }^{11}$ later examined the ground-state energy of the same system and showed that the Hartree-Fock contribution exactly cancels the divergence in the electrostatic contribution. The binding energies of classical systems for various lattice structures are also known. ${ }^{12}$ Quantum Monte Carlo (QMC) simulations of the 2D Bose Coulomb liquid were performed by Magro and Ceperley ${ }^{13}$ motivated by the possibility of superfluidity without a condensate. They show, using an argument by Pitaevskii and Stringari, ${ }^{14}$ that the predominance of long-wavelength plasmons rules out the existence of a condensate. In a recent study, Moudgil et al. ${ }^{15}$ considered a charged Bose fluid within the STLS scheme. The same method was also attempted earlier by Caparica and Hipôlito. ${ }^{16}$ Moudgil et al. ${ }^{15}$ considered the system to be in the condensate phase, and reported numerical instabilities beyond $r_{s} \approx 11$. Despite their assumption of the condensate, their calculated results for the static structure factor compared well with the QMC calculations. ${ }^{13}$ Strepparola and Tosi ${ }^{17}$ showed that the numerical instability mentioned above is an artifact: the STLS equations can be solved for even larger values of $r_{s}$, but the utility of this approach is limited as it does not provide an overall agreement with the $\mathrm{QMC}$ results.

Our aim in this paper is to investigate the ground state properties of 2D charged bosons within the hypernettedchain (HNC) approximation scheme, ${ }^{18}$ which has proved to be rather accurate for 3D and 2D charged Bose systems interacting via the $1 / r$ potential. ${ }^{19} \mathrm{We}$ are motivated by the success of the HNC scheme in correctly describing the properties of charged quantum systems especially in comparison with QMC simulations. ${ }^{20}$ We present a comparative study of the ground-state energy, correlation functions, and plasmon dispersion of $2 \mathrm{D}$ charged bosons to demonstrate that the present approach portrays the static and dynamic properties rather well. The static structure factor develops a peak as the density of the system is lowered, which signals the onset of an ordered phase. Our calculated ground-state energy agrees remarkably well with the available QMC data. ${ }^{13}$ The plasmon energies also indicate a phase transition at low density through dynamical coupling between the plasmon mode and two other collective excitations.

In the rest of this paper, we first give an outline of the HNC formalism and some results based on the RPA and STLS scheme in Sec. II. Our results are presented and discussed in Sec. III. We conclude with a brief summary and some remarks in Sec. IV. 


\section{THEORY}

\section{A. HNC formalism}

We consider a two-dimensional charged Bose gas which interacts via a logarithmic potential $V(r)=-\epsilon \ln (r / L)$, (where $L$ is an arbitrary length scale) obtained from the solution to the 2D Poisson equation. The Hamiltonian of the system can be written as

$$
\mathcal{H}=-\frac{1}{r_{s}^{2}} \sum_{i} \nabla_{i}^{2}-\sum_{i<j} \ln \left(r_{i j}\right)+U_{B}
$$

where $U_{B}$ is a constant energy due to the presence of the neutralizing background, $i$ and $j$ are particle indices, and we have used the length scale $L=r_{s} a$ and energy scale $\epsilon$. The dimensionless parameter $r_{s}$ is defined through $\rho$ $=1 /\left(\pi r_{s}^{2} a^{2}\right)$, in terms of the density $\rho$.

The HNC approximation consists in minimizing the expectation value of the total energy with respect to the variational many-body wave function, assumed to be in the Jastrow-Feenberg form, and using the hypernetted-chain closure to relate the distribution functions to the two-body (or higher) correlation functions. In this work we adopt the socalled HNC/0 approximation (we use the abbreviation HNC) to calculate the ground-state correlation functions and energy, which neglects the three-body correlations or elementary diagrams. The formal structure and details of the variational approach to the many-body problem has been discussed in a number of papers. ${ }^{19,21}$ We briefly present the main ingredients of the calculational scheme.

It is known that in the HNC approximation, ${ }^{19}$ when the elementary diagrams are ignored, the total energy can be written as

$$
E=E_{r}+E_{k},
$$

with

$$
\begin{gathered}
\frac{E_{r}}{N}=\frac{\rho}{2} \int d^{2} r\left[[g(r)-1] V(r)+\frac{\hbar^{2}}{m}|\nabla \sqrt{g(r)}| 2\right], \\
\frac{E_{k}}{N}=-\frac{\hbar^{2}}{8 m \rho} \int \frac{d^{2} q}{(2 \pi)^{2}} q^{2} \frac{[S(q)-1]^{3}}{S(q)},
\end{gathered}
$$

where $S(q)$ and $g(r)$ are the structure factor and pair correlation function, respectively. The first and second term in Eq. (2) are associated with the potential and kinetic energies.

The HNC self-consistent equations can be obtained by minimizing the energy defined in Eq. (2) with respect to $g(r)$ or $S(q)$. The resulting Euler-Lagrange equation yielding the optimized correlation functions ${ }^{22}$ reads

$$
-\frac{\hbar^{2}}{m} \nabla^{2} \sqrt{g(r)}+\left[V(r)+w_{\text {ind }}(r)\right] \sqrt{g(r)}=0,
$$

where $V(r) \sim \ln (r)$ is the bare interaction potential and $w_{\text {ind }}(r)$ is the induced interaction. In the momentum space formulation of this approach, the form of $S(q)$ can be expressed in terms of the particle-hole interaction as ${ }^{19}$

$$
S(q)=\left\{1+\frac{4 m}{\hbar^{2} q^{2}} V_{p-h}(q)\right\}^{-1 / 2},
$$

where the interaction part is given by

$$
V_{p-h}(r)=g(r) V(r)+\frac{\hbar^{2}}{m}|\nabla \sqrt{g(r)}|^{2}+[g(r)-1] w_{\text {ind }}(r),
$$

and the Fourier transform of the induced interaction satisfies the self-consistency relation

$$
w_{\text {ind }}(q)=-\frac{\hbar^{2} q^{2}}{2 m}[S(q)-1]-V_{p-h}(q) .
$$

The particle-hole interaction $V_{p-h}(q)$ here is an effective boson interaction which should not be confused with a similar terminology in Fermi systems. We are following Apaja et $a{ }^{19}{ }^{19}$ in outlying the formal expressions of the HNC theory. One should also note that throughout the calculations we have used the dimensionless Fourier transform as follows:

$$
f(q)=\rho \int d^{2} r f(r) e^{i \mathbf{q} \cdot \mathbf{r}} .
$$

The set of Eqs. (5), (6), and (7) can be solved iteratively for $g(r)$ and $S(q)$. Then, one may go back and calculate the ground state energy using the expression given by Eq. (2).

We note that the logarithmic potential considered in the present application of the HNC scheme presents some numerical problems if the above expressions are used directly. To circumvent these difficulties, we introduce the following transformations in the HNC equations: $V_{p-h}(r) \rightarrow V_{p-h}(r)$ $-V(r)$ and $w_{\text {ind }}(r) \rightarrow w_{\text {ind }}(r)+V(r)$. These give rise to a new set of equations for the static structure factor

$$
S(q)=\left\{1+\frac{4 m}{\hbar^{2} q^{2}}\left[V_{p-h}(q)+V(q)\right]\right\}^{-1 / 2},
$$

and for the effective particle-hole interaction,

$$
V_{p-h}(r)=\frac{\hbar^{2}}{m}|\nabla \sqrt{g(r)}|^{2}+[g(r)-1] w_{\text {ind }}(r),
$$

the induced interaction being still given by Eq. (7). The new set of equations is free from numerical divergence problems, since the Fourier transform of the bare interaction is avoided. In the following section, we present our numerical results for various quantities of interest.

\section{B. RPA and STLS energies}

It would be of interest to compare the results of the HNC approximation with those of the RPA and STLS. Here, we sketch the calculation of ground state energies in the latter approaches. The potential energy per particle is given by

$$
u=-\frac{1}{2}\left[(\gamma-\ln 2)+\int_{0}^{\infty} d q \ln q \frac{d S(q)}{d q}\right],
$$


where $\gamma=-0.577215$ is the Euler-Mascheroni constant. We have used the length and energy scalings introduced earlier. The above expression is obtained by considering a representation of the 2D Coulomb interaction ${ }^{23}$

$$
V(q)=\pi \frac{d}{d s}\left(2^{2-s} \frac{\Gamma(1-s / 2)}{\Gamma(s / 2)} q^{s-2}\right)_{s=0},
$$

$\Gamma(x)$ being the Gamma function, in the potential energy (per particle) formula $u=\frac{1}{2} \Sigma_{k} V(k)[S(k)-1]$, and by integrating by parts.

In the STLS approximation ${ }^{17}$ we have

$$
\frac{d S(q)}{d q}=-r_{s}^{2} S^{3}(q)\left[\frac{S(q)-1}{q^{3}}-8 \frac{1-G(q)}{q^{5}}\right],
$$

where $G(q)$ is the local field factor for exchange and correlation. In particular, the RPA follows when we set $G(q)$ $=0$, and the potential energy per particle is obtained analytically as

$$
u_{\mathrm{RPA}}=-\frac{1}{2}\left[\gamma-\ln 2+\frac{1}{2} \ln r_{s}\right] .
$$

The ground-state energy is calculated through an integral over the coupling strength

$$
E=\frac{2}{r_{s}^{2}} \int_{0}^{r_{s}} d r_{s}^{\prime} r_{s}^{\prime} u\left(r_{s}\right),
$$

which in the RPA yields the analytical result

$$
E_{\mathrm{RPA}}=\frac{1}{8}\left[1-4 \gamma+4 \ln 2-2 \ln r_{s}\right] .
$$

In the case of the STLS approximation, the integrals for $u$ and $E$ need to be computed numerically.

\section{RESULTS AND DISCUSSION}

In this section we present the results of the numerical calculations for static and dynamic properties of the 2D gas of charged bosons. We numerically solve the set of Eqs. (9), (10), and (7) with the repulsive logarithmic potential and find the static structure factor and the pair correlation function, from which all other physical quantities of present interest follow. The principle of our numerics is based on a selfconsistent iteration by initially choosing $V_{p-h}=0$ and $w_{\text {ind }}$ $=0$, and continuing the procedure until a desired accuracy in the converged results is achieved. The calculations are done for different values of the density parameter $r_{s}$.

We first display in Fig. 1 the static structure factor $S(q)$ for 2D charged bosons at $r_{s}=5$ compared with the available QMC data (from Ref. 13) and with STLS results (from Refs. 15 and 16). As may be observed there is good agreement between the HNC and the QMC data at this $r_{s}$ value. Both $S_{\mathrm{HNC}}(q)$ and $S_{\mathrm{QMC}}(q)$ exhibit a peak around $q L \simeq 4$, in contrast to the general shape of $S_{\mathrm{STLS}}(q)$. The long-wavelength behavior $(q \rightarrow 0)$ of $S(q) \sim q^{2}$ smoothly develops in the HNC a peak structure at intermediate $q$ values, whereas the STLS result approaches monotonically the asymptotic limit

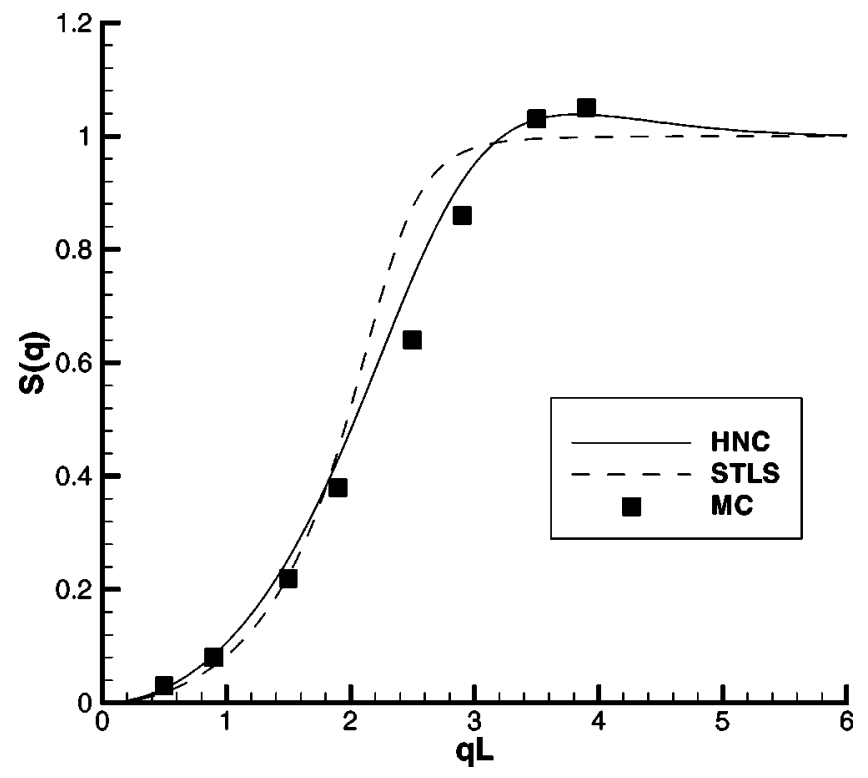

FIG. 1. The static structure factor $S(q)$ versus $q$ (in units of $L^{-1}$ ) for $r_{s}=5$. The solid line shows the present HNC results, which are compared with STLS results (dashed line) and QMC data (squares).

$S(q \rightarrow \infty)=1$. Thus, the HNC approximation provides a better description of the static structure factor of $2 \mathrm{D}$ charged bosons.

The systematic trends in the static structure factor are illustrated in Fig. 2 by giving $S(q)$ for some values of the density parameter. As the density is decreased on increasing $r_{s}$, the correlation effects become stronger and $S(q)$ develops a broad peak around $q L \simeq 4$. The discrepancies between $S_{\mathrm{HNC}}(q)$ and $S_{\mathrm{STLS}}(q)$ become notable for $r_{s} \geq 3$.

The Fourier transform of $S(q)$ yields the pair-distribution function $g(r)$, which is the probability of finding a boson at some distance $r$ if another one is at the origin. It is well

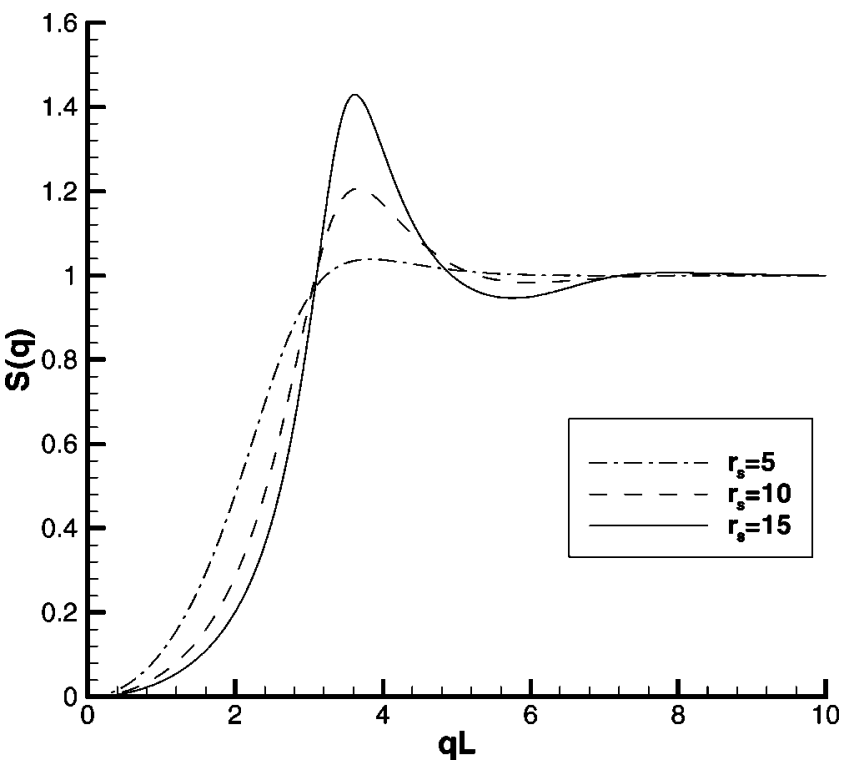

FIG. 2. The static structure factor $S(q)$ in the HNC for $r_{s}=5$ (dotted line), $r_{s}=10$ (dashed line), and $r_{s}=15$ (solid line). 


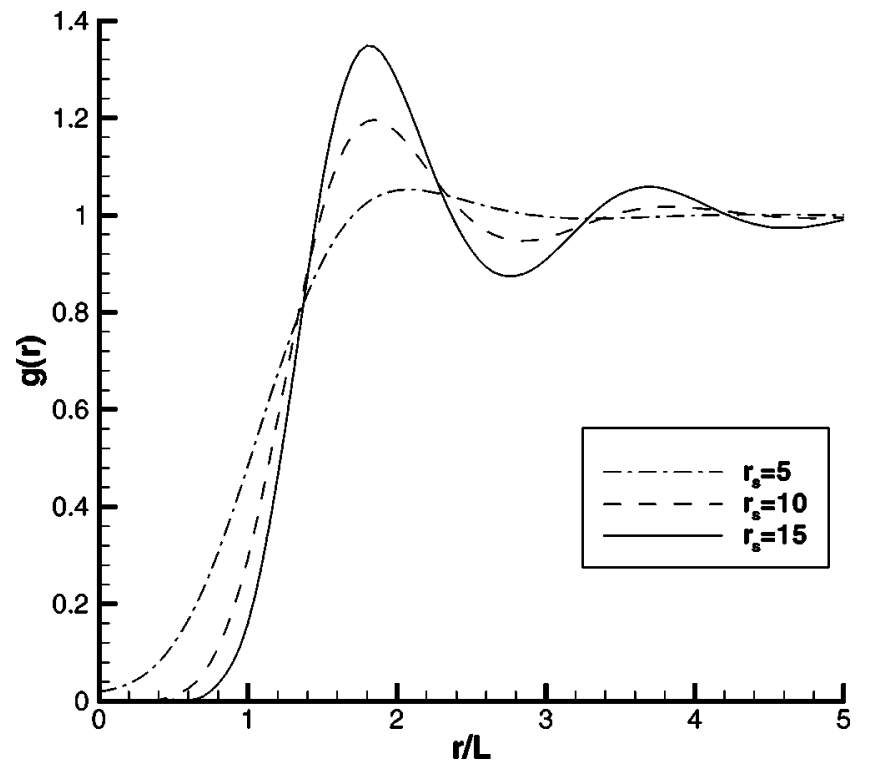

FIG. 3. The pair-distribution function $g(r)$ for various values of $r_{s}$ (given in the legend).

known that the oscillatory behavior of $g(r)$ is a signature of short range order in a fluid. Fig. 3 shows how the oscillatory behavior develops in $g(r)$ with increasing $r_{s}$. Similar trends are also observed within the STLS approximation, ${ }^{15,17}$ but the peak heights there are less pronounced.

The ground-state energy as a function of the density parameter $r_{s}$ is shown in Fig. 4. The HNC results are compared with the QMC data and with the values calculated within the RPA and the STLS. There is very good agreement between the HNC and the QMC results. The STLS energy is also very close to the QMC data, even though the correlation functions within this approximation become inaccurate beyond $r_{s}$ $\approx 5 .{ }^{17}$ Our results for the correlation functions and the ground-state energy of the fluid phase may be useful in

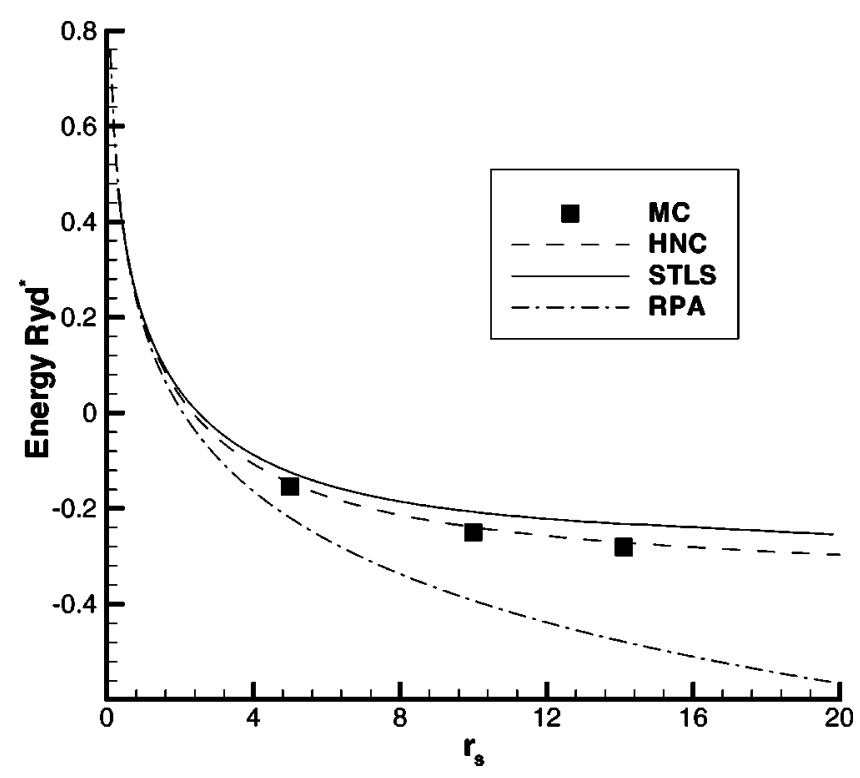

FIG. 4. The ground-state energy $E\left(r_{s}\right)$ per particle within the HNC, RPA, and STLS approximations, compared with QMC data.

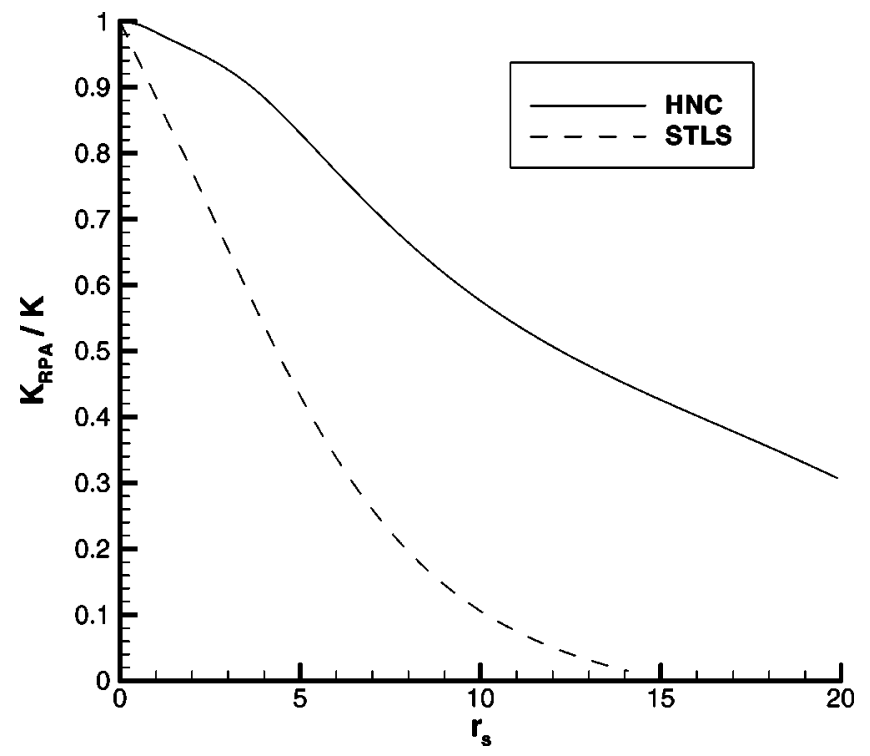

FIG. 5. The inverse compressibility $\kappa_{\mathrm{RPA}} / \kappa$ as a function of $r_{s}$ in HNC and STLS.

studying the transition to the solid phase by the density functional theory of freezing. ${ }^{24}$

The kinetic energy and other thermodynamic quantities of the charged boson fluid are also of interest. An exact relation exists between the pressure and the kinetic energy $P$ $=E_{k} / \pi$, which follows from the virial theorem ${ }^{25}$ The thermodynamic definition of the pressure is $P$ $=-\left(r_{s} / 2 \pi\right) d E / d r_{s}$, and the isothermal compressibility $\kappa$ is given by $1 / \kappa=r_{s}\left(-d E / d r_{s}+r_{s} d^{2} E / d r_{s}^{2}\right) /(4 \pi)$.

In Fig. 5 we show the inverse compressibility $1 / \kappa$ as a function of the density parameter $r_{s}$. The RPA result (in units of $\left.\epsilon / L^{2}\right)$ is a constant, $1 / \kappa_{\mathrm{RPA}}=1 /(8 \pi)$. In this context, we mention that the compressibility calculated from the appropriate sum rule on the static dielectric function does not agree well with the thermodynamic result shown in Fig. 5.

The exchange and correlation effects beyond the RPA are described by the local field factor $G(q)$ (see Ref. 7). The STLS approximation provides a self-consistent way of calculating $G(q)$ within the dielectric formalism. If we identify our $V_{p-h}(q)$ as an effective interaction, we can deduce the corresponding local field factor in the $\mathrm{HNC}$ to read $G_{\mathrm{HNC}}(q)=1-\left(q^{2} / 4 m\right)\left[1 / S^{2}(q)-1\right] / V(q)$. In Fig. 6 we show $G_{\mathrm{HNC}}(q)$ as a function of $q$, for several values of the density parameter. This function exhibits structure which becomes more pronounced with increasing $r_{s}$. In contrast, $G_{\text {STLS }}(q)$ is a monotonic function of $q .{ }^{17}$ The local field factor being greater than unity may be interpreted as the boson liquid becoming unstable against the formation of charge-density wave (CDW) at a certain $r_{s}$ value. Furthermore, the screening properties discussed in the context of STLS approximation would be modified because of the new local-field factors.

Within the same viewpoint, which interprets $V_{p-h}(q)$ as an effective interaction, we can build an approximate model for the dynamic density response function $\chi(q, \omega)$ of the $2 \mathrm{D}$ charged-boson fluid as 


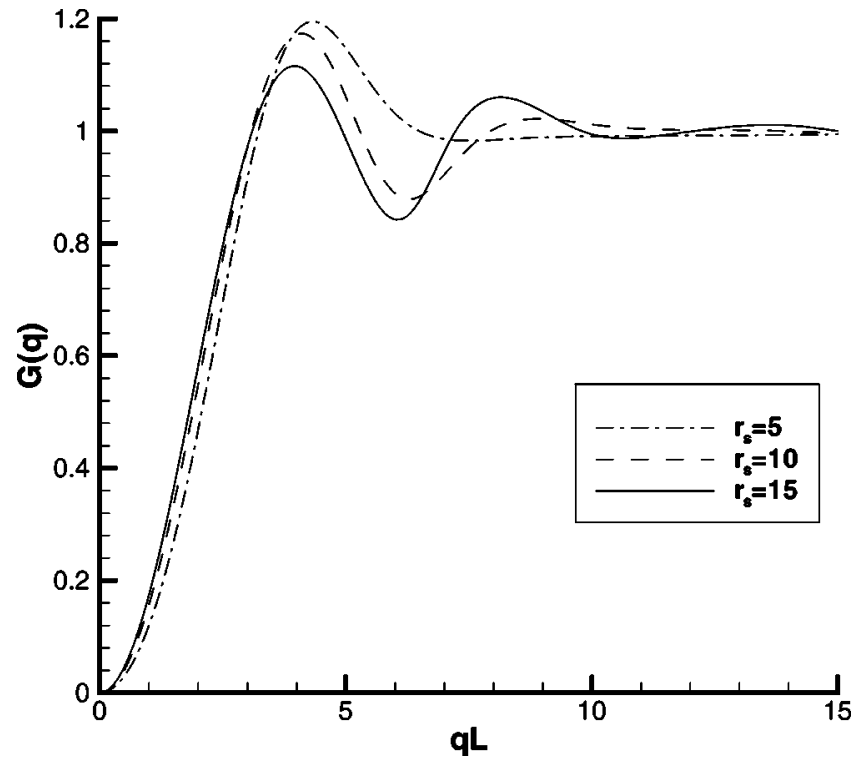

FIG. 6. The local-field correction factor $G(q)$ in HNC for various values of $r_{s}$.

$$
\chi(q, \omega)=\frac{\chi_{0}(q, \omega)}{1-V_{p-h}(q) \chi_{0}(q, \omega)} .
$$

Here, $\chi_{0}(q, \omega)$ is the response function of the noninteracting bosons, $\quad \chi_{0}(q, \omega)=2 \rho\left(q^{2} / 2 m\right) /\left[\omega^{2}-\left(q^{2} / 2 m\right)^{2}+i \eta\right]$. The poles of $\chi(q, \omega)$ in Eq. (17) allow an estimate of the dispersion relation of the plasmon mode, which is shown in Fig. 7 for various values of $r_{s}$.

We see from Fig. 7 that the estimated dispersion curve has a minimum and that the frequency at which the minimum approaches the value $\omega_{p} / 2$ at $q L \approx 3$ for $r_{s} \simeq 10$. A dynamical coupling between the plasma mode and two "rotonic" excitations ${ }^{26}$ becomes important at such values of the coupling strength. It is interesting to note that such a dynamical coupling occurs at a density close to the crystallization density (i.e., $r_{s} \approx 12$ ) reported in QMC simulations. ${ }^{13}$

The nature of the condensate in the system of $2 \mathrm{D}$ charged bosons is also of interest. QMC simulations ${ }^{13}$ sampling the one-body density matrix found that it decays algebraically at long distances indicating that there is no condensate. Within the HNC theory the momentum distribution and condensate fraction can be calculated using the variational ground-state wave functions and optimal correlation functions. ${ }^{27,28}$ These calculations show that it is important to include the elementary diagrams to obtain reasonable estimates for the onebody density matrix and the momentum distribution. We have not considered the elementary diagrams in our version of the HNC approach, therefore, we do not expect the momentum distribution to be represented very accurately. It

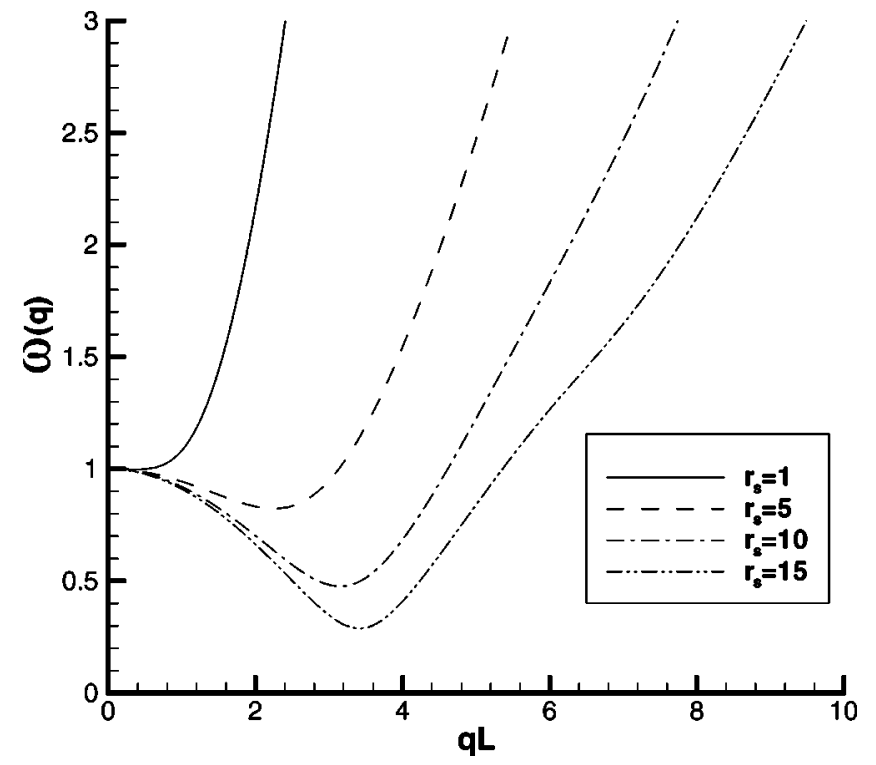

FIG. 7. A sketch of the HNC dispersion relation for the plasmon energy (in units of the plasma frequency $\omega_{p}=\sqrt{2 \pi n e^{2} / m}$ ) at various values of $r_{s}$.

would be interesting to extend our calculations to study the one-body density matrix of charged bosons in more detail, as the QMC simulations ${ }^{13}$ predict interesting behavior for this quantity.

\section{CONCLUSIONS}

In summary, we have calculated the ground-state energy and correlation functions for a two-dimensional charged Bose gas interacting via the logarithmic potential. The HNC approximation yields a good description of this system, when compared with the available QMC results. In particular, the correlation functions signal the onset of an ordered phase at the right density as predicted by the simulations.

The present results provide a significant improvement over the previously considered RPA and STLS approaches. Further improvements through the inclusion of triplet correlations may allow investigations of the dispersion and damping properties of the plasmon and help restore the compressibility sum rule.

\section{ACKNOWLEDGMENTS}

This work is partially supported by the Scientific and Technical Research Council of Turkey (TUBITAK) under Grant No. TBAG-2005 and NATO under Grant No. SfP971970. The work at Scuola Normale Superiore was funded in part by MURST. Two of us (E.S. and M.P.T.) acknowledge useful suggestions from Professor G. Senatore.
${ }^{1}$ T. Ando, A.B. Fowler, and F. Stern, Rev. Mod. Phys. 54, 437 (1982).

${ }^{2}$ Perspectives in Quantum Hall Effects: Novel Quantum Liquids in Low-Dimensional Semiconductor Structures, edited by S.
Das Sarma and A. Pinczuk (Wiley, New York, 1996).

${ }^{3}$ P. Minnhagen, Rev. Mod. Phys. 59, 1001 (1987), and references therein.

${ }^{4}$ A.S. Alexandrov and N.F. Mott, Phys. Rev. Lett. 71, 1075 
(1993); R. Mincas, J. Ranninger, and S. Robaszkiewicz, Rev. Mod. Phys. 62, 113 (1992).

${ }^{5}$ S. Conti, M.L. Chiofalo, and M.P. Tosi, J. Phys.: Condens. Matter 6, 8795 (1994).

${ }^{6}$ R. Calinon, K.I. Golden, G. Kalman, and D. Merlini, Phys. Rev. A 20, 329 (1979); 20, 336 (1979).

${ }^{7}$ K.S. Singwi, M.P. Tosi, R.H. Land, and A. Sjölander, Phys. Rev. 179, 589 (1968); K.S. Singwi and M.P. Tosi, Solid State Phys. 36, 177 (1981).

${ }^{8}$ P. Bakshi, R. Calinon, K.I. Golden, G. Kalman, and D. Merlini, Phys. Rev. A 23, 1915 (1981).

${ }^{9}$ J. Caillol, D. Levesque, J.J. Weiss, and J.-P. Hansen, J. Stat. Phys. 28, 235 (1982); S.W. de Leeuw and J.W. Perram, Physica A 113, 546 (1982); 119, 441 (1983).

${ }^{10}$ J.S. Thakur and K.N. Pathak, J. Phys. C 16, 6551 (1983).

${ }^{11}$ K. Dharamvir and K.N. Pathak, J. Phys.: Condens. Matter 2, 4429 (1990).

${ }^{12}$ R.R. Sari, D. Merlini, and R. Calinon, J. Phys. A 9, 1539 (1976).

${ }^{13}$ W.R. Magro and D.M. Ceperley, Phys. Rev. Lett. 73, 826 (1994).

${ }^{14}$ L. Pitaevskii and S. Stringari, J. Low Temp. Phys. 85, 377 (1991).

${ }^{15}$ R.K. Moudgil, P.K. Ahluwalia, K. Tankeshwar, and K.N. Pathak, Phys. Rev. B 55, 544 (1997).

${ }^{16}$ A.A. Caparica and O. Hipôlito, Phys. Rev. A 32, 560 (1985).

${ }^{17}$ E. Strepparola and M.P. Tosi, Mod. Phys. Lett. B 12, 459 (1998).
${ }^{18}$ E. Feenberg, Theory of Quantum Fluids (Academic Press, New York, 1969).

${ }^{19}$ V. Apaja, J. Halinen, V. Halonen, E. Krotscheck, and M. Saarela, Phys. Rev. B 55, 12925 (1997).

${ }^{20}$ G. Sugiyama, C. Bowen, and B.J. Alder, Phys. Rev. B 46, 13042 (1992); S. Moroni, S. Conti, and M.P. Tosi, ibid. 53, 9688 (1996).

${ }^{21}$ E. Krotscheck and M. Saarela, Phys. Rep. 232, 1 (1993).

${ }^{22}$ See also for original development of the theory, L. Lantto, Nucl. Phys. A238, 297 (1979); R.A. Smith, ibid. A238, 186 (1979).

${ }^{23}$ E.R. Smith, Mol. Phys. 45, 915 (1981).

${ }^{24}$ S. Moroni and G. Senatore, Europhys. Lett. 16, 373 (1991); C.N. Likos, S. Moroni, and G. Senatore, Phys. Rev. B 55, 8867 (1997).

${ }^{25}$ For a 2D system of particles interacting via the $r^{-\alpha}$ law, the virial theorem reads $2 E_{k}+\alpha u=2 P / \rho$, where $P$ is the pressure. For an $\ln r$ interaction $\alpha=0$ and $P=\rho E_{k}$.

${ }^{26}$ H.M. Böhm, S. Conti, and M.P. Tosi, J. Phys.: Condens. Matter 8, 781 (1996); S. Conti, R. Nifosí, and M.P. Tosi, ibid. 9, L475 (1997); R. Nifosí, S. Conti, and M.P. Tosi, Phys. Rev. B 58, 12 758 (1998).

${ }^{27}$ M. Puoskari and A. Kallio, Phys. Rev. B 30, 152 (1984).

${ }^{28}$ E. Manousakis, V.R. Pandharipande, and Q.N. Usmani, Phys. Rev. B 31, 7022 (1985). 